\title{
OPTIMALITY CONDITION AND DUALITY IN MULTI OBJECTIVE PROGRAMMING WITH GENERALIZED $(\varphi, \rho)$-UNIVEXITY.
}

\author{
Deo Brat Ojha \\ R. K. G. I. T., Ghaziabad (U.P.), INDIA \\ Corresponding address: ojhdb@yahoo.co.in \\ Received 1 May, 2010; Revised 11 December, 2010
}

\begin{abstract}
In this paper, we extend the classes of generalized type I vector valued functions introduced by Aghezzaf and Hachimi[1] to generalized univex type I vector-valued functions and consider a multiobjective optimization problem involving generalized type I function with $(\varphi, \rho)$-univexity. A number of Kuhn-Tucker type sufficient optimality conditions are obtained for a feasible solution to be an efficient solution. The Mond-Weir and general Mond-Weir type duality results are also presented.
\end{abstract}

\section{INTRODUCTION}

Rueda et al.[2] obtained optimality and duality results for several mathematical programs by combining the concept of type I functions and univex functions [3]. Mishra[4] obtained optimality. duality and saddle point results for a multiple-objective program by combining the concept of pseudoquasi, type I , quasi-pseudo type I, strictly pseudoquasi, type I and univex functions. Mishra et al.[5] introduce new class of generalized type I univex functions by extending weak strictly pseudoquasi type I, strong pseudoquasi type I etc.Recently Caristi, Ferrara and Stefanescu[6] introduced $(\varphi, \rho)$ invexity.

In this paper, we introduce new class of generalized type I univex functions with $(\varphi, \rho)$ univexity and also studied weak strictly pseudoquasi type I,strong pseudoquasi type I, weak quasistrictly-pseudo type I and weak strictly pseudo type I. In section 2,we introduce some preliminaries. Some sufficient optimality results are established in section 3. A number of duality theorems in the Mond-Weir type are shown in section 4.In section 5. We are giving two results on general Mond-Weir type duality.

\section{PRELIMINARIES}

To compare vectors along the lines of Mangasarian [7],we will distinguish between $\leq$ and $\leqq$ or between $\geq$ and $\geqq$ specifically. $x \in R^{n}, y \in R^{n}, x \leq y \Leftrightarrow x_{i} \leqq y_{i} \forall i=1,2, \ldots \ldots . . n, x \neq y$,

Similarly notations are applied to distinguish between $\geq$ and $\geqq$.

We consider the following multiple objective optimization problem:

(VP) minimize $f(x)=\left(f_{1}(x) \ldots \ldots \ldots . . . f_{p}(x)\right)$ subject to $g(x) \leqq x, x \in X \subseteq R^{n}$.

where $f: X \rightarrow R^{p}$ and $g: X \rightarrow R^{m}$ are differentiable functions and $X \subseteq R^{n}$ is an open set.

Let $\mathrm{X}_{0}$ be the set of all feasible solutions of (VP). We quote some definitions and also give some new ones.

\section{Definition 2.1}

A point $a \in X_{0}$ is said to be an efficient solution of problem (VP) if there exit no $x \in X_{0}$ such that $f(x) \leq f(a), f(x) \neq f(a)$.

\section{Definition 2.2}


A point $a_{a \in X_{0}}$ is said to be a weakly efficient solution of problem (VP) if there is no $x \in X$ such that $f(x)<f(a)$.

\section{Definition 2.3}

A point $a \in X_{0}$ is said to be a properly efficient solution of (VP) if it is efficient and there exist a positive constant $\mathrm{K}$ such that for each $x \in X_{0}$ and for each $i \in\{1,2 \ldots . . p\}$ satisfying $f_{i}(x)<f_{i}(a)$, there exist at least one $i \in\{1,2 \ldots \ldots . p\}$ suchthat $f_{j}\left(a \nmid{ }_{j} f\right.$ and $f_{i}(a)-f_{i}(x) \leq K\left(f_{j}(x)-f_{j}(a)\right)$.

Denoting by WE(VP), E(VP) and PE(VP) the sets of all weakly efficient, efficient and properly efficient solutions of $(\mathrm{VP})$, we have $\mathrm{PE}(\mathrm{VP}) \subseteq \mathrm{E}(\mathrm{VP}) \subseteq \mathrm{WE}(\mathrm{VP})$.

For convenience, let us write the definitions of $(\Phi, \rho)$-univexity on the lines from[1], Let $\varphi: X_{0} \rightarrow R$ be a differentiable function $\left(X_{0} \subseteq R^{n}\right), X \subseteq X_{0}$, and $a \in X_{0}$. An element of all $(\mathrm{n}+1)$ - dimensional Euclidean Space $R^{n+1}$ is represented as the ordered pair ( $\mathrm{z}, \mathrm{r}$ ) with $z \in R^{n}$ and $r \in R, \rho$ is a real number and $\Phi$ is real valued function defined on $X_{0} \times X_{0} \times R^{n+1}$, suchthat $\varphi(x, a,$.$) is convex \quad$ on $\quad R^{n+1}$ and $\Phi(x, a,(0, r)) \geq 0$, for $\quad$ every $(x, a) \in X_{0} \times X_{0}$ and $r \in R_{+} \cdot b_{0}, b_{1}: X \times X \times[0,1] \rightarrow R_{+} \quad b(x, a)=\lim _{\lambda \rightarrow 0} b(x, a, \lambda) \geq 0$, and $\mathrm{b}$ does not depend upon $\lambda$ if the corresponding functions are differentiable. $\psi_{0}, \psi_{1}: R \rightarrow R$ is an $\mathrm{n}$ dimensional vector- valued function.

We assume that $\psi_{0}, \psi_{1}: R \rightarrow R \quad$ satisfying $\quad u \leq 0 \Rightarrow \psi_{0}(u) \leq 0$ and $u \leqq 0 \Rightarrow \psi_{1}(u) \leqq 0$, and $b_{0}(x, a)>0$ and $b_{1}(x, a) \geqq 0$. and $\psi_{0}(\alpha)=-\psi_{0}(\alpha)$ and $\psi_{1}(-\alpha)=-\psi_{1}(\alpha)$.

\section{Example 2.1[6]}

$\min f(x)=x-1$

$g(x)=-x-1 \leq 0, x \in X_{0} \in[1, \infty)$

$\Phi(x, a ;(y, r))=2\left(2^{r}-1\right)|x-a|+\langle y, x-a\rangle$ for $\psi_{0}(x)=x, \psi_{1}(x)=-x, \rho_{1}=\frac{1}{2}($ for $f)$ and $\rho=1($ for $g)$, then this is $(\phi, \rho)$-univex but it is not $(\phi, \rho)$-invex .

\section{Definition 2.4}

The problem(VP) is said to be weak strictly pseudo type I univex at $a \in X_{0}$ if there exit real valued functions $b_{0}, b_{1} \cdot \psi_{0}, \psi_{1}$ and $\rho$ such that

$b_{0}(x, a) \psi_{0}[f(x)-f(a)] \leq 0 \Rightarrow \varphi(x, a,(\nabla f(a), \rho))<0$.

$-b_{1}(x, a) \psi_{1}[g(a)] \leqq 0 \Rightarrow \varphi(x, a,(\nabla g(a), \rho)) \leqq 0$.

for all $x \in X_{0}$ and for all $i=1,2 \ldots \ldots . . p$ and $j=1,2 \ldots \ldots . .$. If (VP) is weakly strictly pseudo type I $(\phi, \rho)$ - univex at each $a \in X,(\mathrm{VP})$ is said to be weak strictly pseudo type I $(\phi, \rho)$-univex on $\mathrm{X}$. 


\section{Remark 2.1[5]}

There exist functions which are weak strictly pseudoquasi type I univex, with respect to $b_{0}=1=b_{1}, \psi_{0}$ and $\psi_{1}$ are identity function on $R$, but not strictly pseudoquasi type I univex, with respect to same $b_{0}, b_{1}, \psi_{0}, \psi_{1}, \rho$.

\section{Definition 2.5.}

The problem (VP) is said to be strong pseudoquasi type I $(\varphi, \rho)$ - univex at $a \in X_{0}$ at if there exit real- valued functions $b_{0}, b_{1} \cdot \psi_{0}, \psi_{1}$ and $\rho$ such that

$b_{0}(x, a) \psi_{0}[f(x)-f(a)] \leq 0 \Rightarrow \phi(x, a,(\nabla f(a), \rho)) \leq 0$.

$-b_{1}(x, a) \psi_{1}[g(a)] \leqq 0 \Rightarrow \phi(x, a,(\nabla g(a), \rho)) \leq 0$.

for all $x \in X_{0}$ and for all $i=\{1,2 \ldots . . P\}$ and $j=\{1,2 \ldots \ldots . m\}$. if (VP) is strong pseudoquasi type $\mathrm{I}(\varphi, \rho)$ univex at each $a \in X,(\mathrm{VP})$ is said to strong pseudoquasi type $\mathrm{I}(\varphi, \rho)$-univex on $\mathrm{X}$.

\section{Remark 2.2[5]}

There exist functions which are strong pseudoquasi type I univex with respect to $b_{0}=1=b_{1}$, $\psi_{0}$ and $\psi_{1}$ are identity function on $R$, but not weak strictly pseudoquasi type I univex with respect to same $b_{0}, b_{1}, \psi_{0}, \psi_{1}, \rho$.

\section{Definition 2.6}

The problem (VP) is weak quasi strictly Pseudo type I $(\varphi, \rho)$ - univex with respect to $b_{0}, b_{1} \cdot \psi_{0}, \psi_{1}$ and $\rho a t a \in X_{0}$ if there exit real-valued functions $b_{0}, b_{1} \cdot \psi_{0}, \psi_{1}$ and $\rho$ such that $b_{0}(x, a) \psi_{0}[f(x)-f(a)] \leq 0 \Rightarrow \phi(x, a,(\nabla f(a), \rho))<0$.

$-b_{1}(x, a) \psi_{1}[g(a)] \leqq 0 \Rightarrow \phi(x, a,(\nabla g(a), \rho))<0$. for $\quad$ all $\quad x \in X_{0} \quad$ and $\quad$ for all $i=\{1,2 \ldots . . p\}$ and $j=\{1,2 \ldots \ldots . . m\}$. If (VP) is weak quasi strictly pseudo type I univex at each $a \in X$, (VP) is said to be weak quasi strictly pseudo type I $(\varphi, \rho)$ - univex on X.

\section{Definition 2.7}

Weak strictly pseudo type I $(\varphi, \rho)$ - univex with respect to $b_{0}, b_{1} \cdot \psi_{0}, \psi_{1}$ and $\rho$ at $a \in X_{0}$ if there exit real-valued functions $b_{0}, b_{1} \cdot \psi_{0}, \psi_{1}$ and $\rho$ such that

$b_{0}(x, a) \psi_{0}[f(x)-f(a)] \leq 0 \Rightarrow \phi(x, a,(\nabla f(a), \rho))<0$.

$-b_{1}(x, a) \psi_{1}[g(a)] \leqq 0 \Rightarrow \phi(x, a,(\nabla g(a), \rho))<0$.

for all $x \in X_{0}$ and for all $i=\{1,2 \ldots \ldots . . p\}$ and $j=\{1,2 \ldots \ldots . m\}$. If (VP) is weak strictly pseudo type I univex at each $a \in X,(\mathrm{VP})$ is said to be weak strictly pseudo type I $(\varphi, \rho)$ - univex on X.

\section{OPTIMALITY CONDITIONS}

In this section, we establish some sufficient optimality condition for an $a \in X_{0}$ to be an efficient solution of problem (VP) under various generalized type I $(\varphi, \rho)$ - univex functions defined in the previous section.

Theorem 3.1 (sufficiency) Suppose that

(i) $a \in X_{0}$ (ii) There exist $\tau^{0} \in R^{p}, \tau^{0}>0, \lambda \in R^{m}$ and $\lambda^{0} \geqq 0$ Such that

(a) $\tau^{0} \nabla f(a)+\lambda^{0} \nabla g(a)=0$ (b) $\lambda^{0} g(a)=0$ (c) $\tau^{0} e=1$, where $e=(1, \ldots \ldots 1)^{T} \in R^{P}$; 
(iii)The problem (VP) is strong pseudoquasi type I $(\varphi, \rho)$ - univex at $a \in X_{0}$ with respect to some $b_{0}, b_{1} \cdot \psi_{0}, \psi_{1}$ and $\rho$ for all feasible $x$. then a is an efficient solution to (VP).

\section{Proof}

Suppose contrary to the result that a is not an efficient solution to (VP). Then there exists a feasible solution $x$ to (VP) such that $f(x) \leq f(a)$.

By the properties of $b_{0}$ and $\psi_{0}$ and the above inequality, we have $b_{0}(x, a) \psi_{0}[f(x)-f(a)] \leq 0(1)$

By the feasibility of a, we have $-\lambda^{0} g(a) \leq 0$

By the properties of $b_{1}$ and $\psi_{1}$ and the above inequality,

we have $-b_{1}(x, a) \psi_{1}\left[\lambda^{0} g(a)\right] \leqq 0$

By inequalities (1) and (2) and condition (iii), we have

$\phi(x, a ;(\nabla f(a), \rho)) \leq 0$ and $\phi\left(x, a ;\left(\lambda^{0} \nabla g(a), \rho\right)\right) \leq 0$, Since $\tau^{0}>0, \quad$ the above inequalities give $\phi\left(x, a ;\left(\tau^{0} \nabla f(a)+\lambda^{0} \nabla g(a), \rho\right)\right)<0$

which contradict condition (iii). This completes the proof.

Theorem 3.2 (sufficiency) Suppose that

(i) $a \in X_{0}$ (ii) There exist $\tau^{0} \in R^{p}, \tau^{0} \geq 0, \lambda \in R^{m}$ and $\lambda^{0} \geqq 0$ Such that

(a) $\tau^{0} \nabla f(a)+\lambda^{0} \nabla g(a)=0$ (b) $\lambda^{0} g(a)=0$ (c) $\tau^{0} e=1$, where $e=(1, \ldots \ldots . .1)^{T} \in R^{P}$;

(iii) The problem (VP) is weak strictly pseudoquasi type I $(\varphi, \rho)$ - univex at $a \in X_{0}$ with respect to some $b_{0}, b_{1} \cdot \psi_{0}, \psi_{1}$ and $\rho$ for all feasible $x$. then a is an efficient solution to (VP).

\section{Proof}

Suppose contrary to the result that a is not an efficient solution to (VP). Then there exists a feasible solution $x$ to (VP) such that $f(x) \leq f(a)$.

By the property of $b_{0}$ and $\psi_{0}$ and the above inequality, we get (1). By the feasibility of a the properties of $b_{1}$ and $\psi_{1}$ and the condition (iii), we have

$\phi(x, a ;(\nabla f(a), \rho))<0$ and $\phi\left(x, a ;\left(\lambda^{0} \nabla g(a), \rho\right)\right) \leq 0$, Since $\tau^{0} \geq 0$, the above inequalities give $\phi\left(x, a ;\left(\tau^{0} \nabla f(a)+\lambda^{0} \nabla g(a), \rho\right)\right)<0$

which contradicts (iii). This completes the proof.

Theorem 3.3 (sufficiency) Suppose that

(i) $a \in X_{0}$ (ii) There exist $\tau^{0} \in R^{p}, \tau^{0} \geqq 0, \lambda \in R^{m}$ and $\lambda^{0} \geqq 0$ Such that

(a) $\tau^{0} \nabla f(a)+\lambda^{0} \nabla g(a)=0$ (b) $\lambda^{0} g(a)=0$ (c) $\tau^{0} e=1$, where $e=(1, \ldots . .1)^{T} \in R^{P}$;

(iii) The problem (VP) is weak strictly pseudo type I $(\varphi, \rho)$ - univex at $a \in X_{0}$ with respect to some $b_{0}, b_{1} \cdot \psi_{0}, \psi_{1}$ and $\rho$ for all feasible $x$, then a is an efficient solution to (VP).

\section{Proof}

Suppose contrary to the result that a is not an efficient solution to (VP). Then there exists a feasible solution $x$ to (VP) such that $f(x) \leq f(a)$.

By the property of $b_{0}$ and $\psi_{0}$ and the above inequality, we get (1). By the feasibility of a and properties of $b_{1}$ and $\psi_{1}$ we get (2). By inequalities (1) and (2) and condition (iii), we have $\phi(x, a ;(\nabla f(a), \rho))<0$ and $\phi\left(x, a ;\left(\lambda^{0} \nabla g(a), \rho\right)\right)<0$, Since $\tau^{0} \geqq 0, \quad$ the above inequalities give $\phi\left(x, a ;\left(\tau^{0} \nabla f(a)+\lambda^{0} \nabla g(a), \rho\right)\right)<0 \quad$ which contradicts (iii). This completes the proof. 


\section{MOND-WEIR TYPE DUALITY}

In this section, we present some weak and strong duality theorems for (VP) and the following Mond-Weir dual problem suggested by Egudo[7]:

(MWD) Maximize f(y)

$$
\text { Subject to } \tau \nabla f(y)+\lambda \nabla g(y)=0
$$

$$
\lambda \nabla g(y) \geqq 0
$$

$\lambda \geqq 0, \tau \geqq 0$ and $\tau e=1$, where $e=(1, \ldots \ldots .1)^{T} \in R^{P}$, Denote by $\mathrm{Y}^{0}$ the set of all the feasible solutions of problem (MWD), i.e. ,

$\mathrm{Y}^{0}=\left\{(y, \tau, \lambda) ; \tau \nabla f(y)+\lambda \nabla g(y)=0, \lambda \nabla g(y) \geqq 0, \tau \in R^{p}, \lambda \in R^{m}, \lambda \geqq 0\right\}$

Theorem 4.1 ( Weak duality ) Suppose that

(i) $x \in X_{0}$ (ii) $(y, \tau, \lambda) \in \mathrm{Y}^{0}$ and $\tau>0$;

(iii) Problem (VP) is strong pseudoquasi type I $(\varphi, \rho)$ - univex at y with respect to some $b_{0}, b_{1} \cdot \psi_{0}, \psi_{1}$ and $\rho$ then $f(x) \not f(y)$.

\section{Proof}

Suppose contrary to the result i.e, $f(x) \leq f(y)$.

By the property of $b_{0}$ and $\psi_{0}$ and the above inequality, we have $b_{0}(x, a) \psi_{0}[f(x)-f(y)] \leq 0$

By the feasibility of $(y, \tau, \lambda)$, we have $-\lambda^{0} g(y) \leqq 0$, By the properties of $b_{1}$ and $\psi_{1}$ we get $-b_{1}(x, a) \psi_{1}[\lambda g(y)] \leqq 0$

By the inequalities (4) and (5) and condition (iii), we have $\phi(x, y ;(\nabla f(y), \rho)) \leq 0$ and $\phi(x, y ;(\lambda \nabla g(y), \rho)) \leq 0$, Since $\tau>0$, the above inequalities give $\phi(x, y ;(\tau \nabla f(y)+\lambda \nabla g(y), \rho))<0$, which contradicts (iii). This completes the proof.

Theorem 4.2 ( Weak duality) suppose that

(i) $x \in X_{0}$ (ii) $(y, \tau, \lambda) \in \mathrm{Y}^{0}$ and $\tau^{0} \geq 0$;

(iii) Problem (VP) is weak strictly pseudoquasi type I $(\varphi, \rho)$ - univex at y with respect to some $b_{0}, b_{1} \cdot \psi_{0}, \psi_{1}$ and $\rho$ then $f(x) \not \leq f(y)$.

\section{Proof}

Suppose contrary to the result i.e, $f(x) \leq f(y)$. By the properties of $b_{0}$ and $\psi_{0}$ and the above inequality, we get (4). By the feasibility of $(y, \tau, \lambda)$, and properties of $b_{1}$ and $\psi_{1}$ we get (5). By the inequalities (4) and (5) and condition (iii), we have $\phi(x, y ;(\nabla f(y), \rho))<0$ and $\phi(x, y ;(\lambda \nabla g(y), \rho)) \leq 0$, Since $\tau^{0} \geq 0$, the above inequalities give, $\varphi\left(x, y ;\left(\tau^{0} \nabla f(y)+\lambda \nabla g(y), \rho\right)\right)<0$, which contradicts (iii). This completes the proof .

Theorem 4.3 ( Weak duality ) suppose that

(i) $x \in X_{0}$ (ii) $(y, \tau, \lambda) \in \mathrm{Y}^{0}$;

(iii) Problem (VP) is weak strictly pseudo type I $(\varphi, \rho)$ - univex at y with respect to some $b_{0}, b_{1} \cdot \psi_{0}, \psi_{1}$ and $\rho$ then $f(x) \leq f(y)$.

\section{Proof}

Suppose contrary to the result, i.e., $f(x) \leq f(y)$. By the properties of $b_{0}, \psi_{0}$ and the above inequality, we get (4), and the feasibility of $(y, \tau, \lambda)$ and properties of $b_{1}$ and $\psi_{1}$ we get (5).By 
the inequalities (4) and (5) and condition (iii), we have $\phi(x, y ;(\nabla f(y), \rho))<0$ and $\phi(x, y ;(\nabla g(y), \rho))<0$. Which contradicts condition (iii). This completes the proof.

Theorem4.4 ( Strong duality ). Let $\mathrm{z}$ be an efficient solution for (VP) and $\mathrm{z}$ satisfies a constraint qualification for (VP) in Marusciac [8]. Then there exist $b \in R^{p}$ and $c \in R^{m}$ such that $(\mathrm{z}, \mathrm{b}, \mathrm{c})$ is feasible for (MWD). If any of the weak duality in theorems 4.1-4.3 also holds. Then $(\mathrm{z}, \mathrm{b}, \mathrm{c})$ is efficient solution (MWD).

\section{Proof}

Since $\mathrm{z}$ is efficient for (VP) and satisfies the constraint qualification for (VP), then from the Kuhn-Tucker necessary optimality condition, we obtain $\mathrm{b}>0$ and $c \geqq 0$ such that $b \nabla f(z)+c \nabla g(z)=0, c g(z)=0$, the vector $\mathrm{b}$ may be normalized according to be $=1$. $\mathrm{b}>0$, which gives that the triple $(\mathrm{z}, \mathrm{b}, \mathrm{c})$ is feasible for (MWD). The efficiency of $(\mathrm{z}, \mathrm{b}, \mathrm{c})$ for (MWD) follows from weak duality theorem. Thus completes the proof.

\section{GENERAL MOND-WEIR TYPE DUALITY}

In this section, we consider a general Mond-Weir type of dual problem to (VP) establish weak and strong duality theorems under some mild assumption. We consider the following general Mond-Weir type dual problem:

(GMWD) Maximize $\mathrm{f}(\mathrm{y})+\lambda_{\mathrm{j}_{0}} g_{\mathrm{j}_{0}}(y) e$

$$
\begin{aligned}
& \text { Subject to } \tau \nabla f(y)+\lambda \nabla g(y)=0 \\
& \lambda_{\mathrm{j}_{\mathrm{q}}} g_{\mathrm{j}_{\mathrm{q}}} \geqq 0,1 \leq q \leqq r
\end{aligned}
$$

$\lambda \geqq 0, \tau \geqq 0$ and $\tau e=1$, where $e=(1, \ldots \ldots . .1)^{T} \in R^{P}, J_{q}, 1 \leq q \leq r$, are partitions of the set $\mathrm{N}$.

Theorem 5.1 ( Weak duality ) suppose that for all feasible $x$ for (VP) and for all feasible $(y, \tau, \lambda)$ for (GMWD):

(a) $\tau>0$ and (f $\left.+\lambda_{\mathrm{j}_{0}} g_{\mathrm{j}_{0}}() e,. \lambda j_{q} g j_{q}().\right)$ is pseudoquasi type I $(\varphi, \rho)$-univex at y for each $\mathrm{q}$. $1 \leq q \leqq r$ with respect to some $b_{0}, b_{1} \cdot \psi_{0}, \psi_{1}$ and $\rho$;

(b) (f $\left.+\lambda_{\mathrm{j}_{0}} g_{\mathrm{j}_{0}}() e,. \lambda j_{q} g j_{q}().\right)$ is weak strictly pseudoquasi type I $(\varphi, \rho)$-univex at y for each $\mathrm{q}$. $1 \leq q \leq r$ with respect to some $b_{0}, b_{1} \cdot \psi_{0}, \psi_{1}$ and $\rho$;

(c) $\left(\mathrm{f}+\lambda_{\mathrm{j}_{0}} g_{\mathrm{j}_{0}}() e,. \lambda j_{q} g j_{q}().\right)$ is weak strictly pseudo type I $(\varphi, \rho)$-univex at y for each $\mathrm{q}$, $1 \leq q \leq r$ with respect to some $b_{0}, b_{1} \cdot \psi_{0}, \psi_{1}$ and $\rho$; then $f(x) \leq f(y) .+\lambda j_{0} g j_{0}(y) e$.

Proof : Suppose contrary to the result. Thus, we have $f(x) \nless f(y) .+\lambda j_{0} g j_{0}(y) e$.

Since $x$ is feasible for (VP) and $\lambda \geqq 0$, the above inequality implies that $f(x)+\lambda J_{0} g J_{0}(x) e . \leq f(y)+\lambda J_{0} g J_{0}(y) e$.

By the feasibility of $(y, \tau, \lambda)$ inequality (7) gives

$-\lambda J_{q} g J_{q}(y) \leqq 0,1 \leqq q \leqq r$.

Since $\psi_{0}$ and $\psi_{1}$ are increasing, from (8) and (9), we have

$$
\begin{aligned}
& b_{0}(x, y) \psi_{0}\left\{\left(f(x)+\lambda J_{0} g J_{0}(x) e-f(y)+\lambda J_{0} g J_{0}(y) e \leq 0\right.\right. \\
& -b_{1}(x, y) \psi_{1}\left\{\lambda J_{q} g J_{q}(y)\right\} \leqq 0,1 \leqq q \leqq r .
\end{aligned}
$$


By condition (a), from (10) and (11), we have

$\phi\left(x, y ;\left(\nabla f(y)+\lambda J_{0} g J_{0}(y) e, \rho\right)\right) \leq 0$

$\phi\left(x, y ;\left(\lambda J_{q} \nabla g J_{q}(y) e, \rho\right)\right) \leqq 0,1 \leqq q \leqq r$

Since, $\tau>0$ the above inequalities give

$\phi\left(x, y ;\left(\tau \nabla f(y)+\sum_{q=0}^{r} \lambda \nabla J_{q} g J_{q}(y), \rho\right)\right)<0$

Since $J_{q}, 0 \leqq q \leqq r$ are partitions of the set $\mathrm{N},(12)$ is equivalent to

$\phi(x, y ;(\tau \nabla f(y)+\lambda \nabla g(y), \rho))<0$

which contradicts (6), By condition (b), from (10) and (11), we have

$\phi\left(x, y ;\left(\nabla f(y)+\lambda J_{0} g J_{0}(y) e, \rho\right)\right)<0$,

$\phi\left(x, y ;\left(\lambda J_{q} \nabla g J_{q}(y), \rho\right)\right) \leqq 0,1 \leqq q \leqq r$.

Since, $\tau \geq 0$, the above inequalities give (12), which again contradicts (6). By condition (c) ,(10) and (11), we have,

$\phi\left(x, y ;\left(\nabla f(y)+\lambda J_{0} g J_{0}(y) e, \rho\right)\right)<0, \phi\left(x, y ;\left(\lambda J_{q} \nabla g J_{q}(y), \rho\right)\right)<0,1 \leqq q \leqq r$. Since, $\tau \geqq 0, \quad$ the above inequalities give (12), which again contradicts (6). This completes the proof.

Theorem 5.2 (strong duality) let $\mathrm{z}$ be an efficient solution for (VP) and $\mathrm{z}$ satisfies a constraint qualification for (VP). Then there exist $b \in R^{p}$ and $c \in R^{m}$ suchthat $(z, b, c)$ is feasible for (GMWD). If any of the weak duality in theorem 5.1 holds, then $(z, b, c)$ is an efficient solution for (GMWD).

\section{Proof}

Since $\mathrm{z}$ is efficient for (VP) and satisfies a generalized constraint qualification, by the KuhnTucker necessary condition (see Maeda[11]),there exist $\mathrm{b}>0$ and $c \geqq 0$ such that $b \nabla f(z)+c \nabla g(z)=0, c_{\mathrm{i}} g_{\mathrm{i}}(z)=0,1 \leqq i \leqq p$. The vector $\mathrm{b}$ may be normalized according to be $=1$, b> 0 , which gives that the triplet $(z, b, c)$ is feasible for (GMWD). The efficiency follows from the weak duality in theorem 5.1. this completes the proof.

\section{CONCLUSION}

In this paper, we have extended the corresponding results of Mishra [9, 5], Aghezzaf and Hachimi [1], Ferrara and Stefanescu [10] to a wider class of functions.

\section{REFERENCES}

[1] Aghezzaf B \& Hachimi M, Generalized invexity and duality in multi objective programming problems, J. Global optim. 18(2000)91.

[2] Rueda N G, Hanson M A \& Singh C, Optimality and duality with generalized convexity. J. Optim. Theory Appl. 86(1995) 491.

[3] Bector C R, Suneja S K \& Gupta S, Univex functions and univex nonlinear programming in: Proceedings of the Administrative Sciences Association of Canada, (1992), 115 . 
[4] Mishra S K, On multiple-objective optimization with generalized univexity, J. Math . Anal. Appl. 224(1998) 131.

[5] Mishra S K, Ywang S \& Lai K K, Optimality and duality multiple-objective optimization under generalized type I univexity. J. Math. Anal. Appl. 303(2005) 315.

[6] Caristi G, Ferrara M, \& Stefanescu A, Mathematical programming with $(\varphi, \rho)$ invexity in: Igor, V., Konnov, Dinh, the Luc, Alexander, M., Rubinov, (eds), Generalized convexity and Related topics, lecture notes in Economics and Mathematical system, Vol.583. Springer, 2006, 167.

[7] Egudo R R, Efficiency and generalized convex duality for multi objective programs, $J$. Math. Anal. Appl. 138(1989) 84.

[8] Marusciac I, On Fritz John Optimality criterion in multi objective optimization, Anal. Numer, Theorie Approx. 11(1982) 109.

[9] Mishra S K, V-invex functions and applications to multiple-objective programming problems, Ph. D. Thesis, Banaras Hindu university, Varanasi, India,1995.

[10] Ferrara M \& Stefanescu M V, Optimality conditions and duality in Multi objective programming with $(\varphi, \rho)$ - invexity, Yugoslav J. Operations Research, 18(2008) 2, 153.

[11] Maeda T, Constraint qualification in multiobjective optimization problems: differentiable case, J. Optim. Theory Appl. 80(1994)483. 